\title{
ON NORMAL METRICS, AND A THEOREM OF COHN-VOSSEN
}

\author{
BY ROBERT FINN
}

Communicated by S. S. Chern, July 10, 1964

1. Let $\mathrm{g}$ denote the domain consisting of the complex $z$-plane with $n+1$ points $p_{1}, \cdots, p_{n}, p_{0}=\infty$ deleted. Let $\mu(E)$ be a measure over $\mathcal{G}$, of finite total variation. Let

$$
u(x, y)=\int_{\mathcal{G}} \log \left|1-\frac{z}{\zeta}\right| d \mu_{\zeta}+h(z),
$$

where $h(z)$ is harmonic in $\mathrm{g}$. The conformal metric $e^{u(z)}|d z|$ will be said to be normal in $\mathrm{G}$, provided $h(z)$ has the form

$$
h(z)=\sum_{1}^{n} \beta_{j} \log \left|z-p_{j}\right|+\text { const. }
$$

The metric is said to be complete if any path tending to one of the $\left\{p_{j}\right\}$ has infinite length. The curvatura integra is $C=-2 \pi \int_{g} d \mu_{\zeta}$, and the flux at $p_{j}$ is defined by

$$
\Phi_{j}=\lim _{\gamma_{j} \rightarrow p_{j}} \frac{1}{2 \pi} \oint_{\gamma_{j}} \frac{\partial u}{\partial n}|d z|,
$$

for curves $\gamma_{j}$ enclosing $p_{j}$.

Let $\Gamma_{j}, \gamma_{j}$ be concentric circumferences surrounding $p_{j}$, and let $Q_{j}(\Gamma, \gamma)$ be the area of the enclosed annulus in the given metric. Let $\mathcal{L}_{j}(\gamma)$ be the length of $\gamma_{j}$.

Theorem. If the metric is complete, then $\Phi_{0} \geqq-1, \Phi_{j} \geqq 1, j>0 .{ }^{1}$ The quantities

$$
\nu_{j}=\lim _{\gamma_{j} \rightarrow p_{j}} \frac{\mathfrak{L}_{j}(\gamma)}{4 \pi Q_{j}(\Gamma, \gamma)}
$$

exist and are finite for each $j$, and $\nu_{0}=\Phi_{0}+1, \nu_{j}=\Phi_{j}-1, j>0$. There holds

$$
C=2 \pi\left(x-\sum_{0}^{n} \nu_{j}\right)
$$

where $\chi=1-n$ is the Euler characteristic of $\mathrm{g}$.

${ }^{1}$ This assertion follows alternatively from the work of Huber [2]. The demonstrations differ. 
This result contains, for the case considered, a theorem of CohnVossen [1] (see also Huber [2]), according to which $C \leqq 2 \pi \chi$. Cases for which equality holds are easily constructed, but one can also give examples for which the $\left\{\nu_{j}\right\}$ are arbitrarily large.

Asymptotic estimates for $\mathfrak{L}_{j}(\gamma)$ and $Q_{j}(\Gamma, \gamma)$ are given. The curves $\left\{\gamma_{j}\right\}$ are shown to behave as approximate geodesic circles in the metric, thus permitting a posteriori an interpretation of the result in terms of the explicitly given geometry. The asymptotic lengths of the radial segments emanating from the $\left\{p_{j}\right\}$ are also estimated, and these curves are shown to approximate geodesics in the metric.

2. Let $S$ be a finitely connected, open Riemann surface on which a conformal metric $e^{u(z)}|d z|$ is defined, and suppose the curvatura integra associated with the metric is finite. Suppose in addition that the region of positive curvature has compact support on $\delta$.

THEOREM. If the metric is complete, then a doubly connected neighborhood of each boundary component can be mapped conformally onto the exterior of the unit disc. Defining $\Gamma_{j}, \gamma_{j}$ as above, the limit

$$
\nu_{j}=\lim _{\gamma_{j} \rightarrow \infty} \frac{\mathfrak{L}_{j}(\gamma)}{4 \pi Q_{j}(\Gamma, \gamma)}
$$

exists for each such mapping, and $\nu_{j}=\Phi_{j}+1$. There holds

$$
C=2 \pi\left(\chi-\sum_{0}^{n} \nu_{j}\right) \text {. }
$$

The essential step in the proof consists in reducing the problem to the study of metrics, whose local structure is normal.

The reader will very likely share my discomfort over the hypothesis that the region $K>0$ has compact support. I have been unable to prove the result under significantly weaker assumptions.

\section{REFERENCES}

1. S. Cohn-Vossen, Kürzeste Wege und Totalkrümmung auf Flächen, Compositio Math. 2 (1935), 69-133.

2. A. Huber, On subharmonic functions and differential geometry in the large, Comment. Math. Helv. 32 (1957), 13-72.

StANFORd UNIVERSITY 